Pontifícia Universidade $C_{\text {atólica }}$

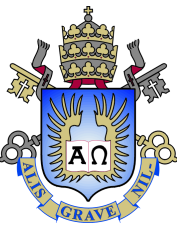

Gabriel de Lima Monteiro

\title{
Soluções Fracas de Equações Diferenciais Elípticas de Segunda Ordem
}

Dissertação de Mestrado

Dissertação apresentada como requisito parcial para obtenção do grau de Mestre pelo Programa de Pós-graduação em Matemática do Departamento de Matemática do Centro Técnico Científico da PUC-Rio.

Orientador: Prof. Boyan Slavchev Sirakov 


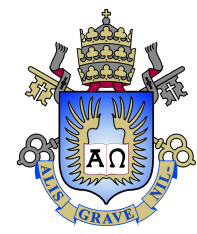

Gabriel de Lima Monteiro

\title{
Soluções Fracas de Equações Diferenciais Elípticas de Segunda Ordem
}

Dissertação apresentada como requisito parcial para obtenção do grau de Mestre pelo Programa de Pós-graduação em Matemática do Departamento de Matemática do Centro Técnico Científico da PUC-Rio. Aprovada pela Comissão Examinadora abaixo assinada.

\author{
Prof. Boyan Slavchev Sirakov \\ Orientador \\ Departamento de Matemática - PUC-Rio
}

Prof. Edgard Almeida Pimentel

Departamento de Matemática - PUC-Rio

Prof. Rubens Luis Pinto Gurgel do Amaral Universidade Federal Fluminense - UFF

Prof. Márcio da Silveira Carvalho

Coordenador Setorial do Centro Técnico Científico - PUC-Rio 
Todos os direitos reservados. É proibida a reprodução total ou parcial do trabalho sem autorização da universidade, do autor e do orientador.

\section{Gabriel de Lima Monteiro}

Graduou-se em Física pela Universidade Federal Fluminense (Niterói, Rio de Janeiro)

Ficha Catalográfica

de Lima Monteiro, Gabriel

Soluções Fracas de Equações Diferenciais Elípticas de Segunda Ordem / Gabriel de Lima Monteiro; orientador: Boyan Slavchev Sirakov. - Rio de janeiro: PUC-Rio, Departamento de Matemática , 2018.

v., 35 f: il. color. ; $30 \mathrm{~cm}$

Dissertação (mestrado) - Pontifícia Universidade Católica do Rio de Janeiro, Departamento de Matemática .

Inclui bibliografia

1. Matemática - Teses. 2. Soluções Fracas - Teses. 3. Soluções Fracas;. 4. Equações Diferenciais Parciais;. 5. Espaços de Sobolev;. I. Slavchev Sirakov, Boyan. II. Pontifícia Universidade Católica do Rio de Janeiro. Departamento de Matemática . III. Título. 


\section{Agradecimentos}

Gostaria de agradecer a meus pais João e Regina pelo suporte e apoio que sempre me deram, a Izabela Hammershlag pelo amor e companheirismo. Agradeço também o meu orientador Prof Boyan Sirakov, os membros desta banca, os professores do departamento de matemática da PUC-Rio, o corpo administrativo do departamento e aos amigos da pós graduação, em especial David e João Gabriel.

Por último, à PUC-Rio pela bolsa de isenção de mensalidades do mestrado. 


\section{Resumo}

de Lima Monteiro, Gabriel; Slavchev Sirakov, Boyan. Soluções Fracas de Equações Diferenciais Elípticas de Segunda Ordem. Rio de Janeiro, 2018. 35p. Dissertação de Mestrado - Departamento de Matemática , Pontifícia Universidade Católica do Rio de Janeiro.

Esse trabalho tem como objetivo ser uma introdução ao estudo da existência e unicidade de soluções fracas para equações diferenciais parciais elípticas. Começamos definindo o espaço de Sobolev para, a partir da definição, provarmos algumas propriedades básicas que nos ajudarão no estudo das equações diferenciais parciais elípticas. Finalizamos com o desenvolvimento do Teorema de Lax-Milgram e de Stampacchia que permitirão o uso de técnicas de Análise Funcional para estudarmos alguns exemplos de equações elípticas.

\section{Palavras-chave}

Soluções Fracas; Equações Diferenciais Parciais; Espaços de Sobolev; 


\section{Abstract}

de Lima Monteiro, Gabriel; Slavchev Sirakov, Boyan (Advisor). Weak Solutions for Elliptic Partial Differential Equations of Second Order. Rio de Janeiro, 2018. 35p. Dissertação de mestrado - Departamento de Matemática , Pontifícia Universidade Católica do Rio de Janeiro.

This dissertation aims to be an introduction to the study of the existence and uniqueness of weak solutions for elliptic partial differential equations. We begin by defining the Sobolev spaces and proving some basics properties that will assist in the study of the elliptical equations. Lastly, we develop the Theorems of Lax-Milgram and Stampacchia that allow the use of Functional Analysis for the studying of some examples of elliptic equations.

\section{Keywords}

Weak Solutions; Partial Differential Equations; Sobolev Spaces; 


\section{Sumário}

1 Introdução $\quad 9$

2 Espaços de Sobolev $\quad 11$

2.1 Definições 11

2.2 Propriedades dos Espaços de Sobolev 13

$\begin{array}{ll}2.3 \text { Desigualdades de Sobolev } & 16\end{array}$

$\begin{array}{ll}\text { 2.3.1 Gagliardo-Nirenberg-Sobolev } & 16\end{array}$

$\begin{array}{ll}2.3 .2 n=p & 18\end{array}$

$\begin{array}{ll}\text { 2.3.3 Morrey } & 19\end{array}$

2.4 Espaço $W_{0}^{1, p} \quad 22$

2.5 Espaços de Sobolev e Hilbert 23

3 Soluções Fracas $\quad \mathbf{2 6}$

$\begin{array}{lll}3.1 & \text { O Teorema de Lax-Milgram } & 26\end{array}$

$\begin{array}{lll}3.2 & \text { Equações } & 27\end{array}$

$\begin{array}{ll}\text { Referências bibliográficas } & 34\end{array}$

$\begin{array}{ll}\text { A Desigualdades } & 35\end{array}$ 


\section{Notações}

$\begin{array}{ll}\Omega & \text { Conjunto aberto e limitado } \\ (u, v) & \text { Produto interno de u,v } \\ E^{*} & \text { Espaço dual de } E \\ \|u\|_{p} & \text { Norma de u em } L^{p}(\Omega) \\ \langle,\rangle & \text { Produto interno para dualidade E e } E^{*} \\ H & \text { Espaço de Hilbert } \\ D_{i} u=\frac{\partial u}{\partial x_{i}} & \\ D u=\left(D_{1} u, \ldots, D_{n} u\right) & \end{array}$




\section{1 \\ Introdução}

Equações diferenciais parciais são equações que envolvem uma função desconhecida, de duas ou mais variáveis, e suas derivadas parciais. Por sua vez, equações diferenciais parciais de segunda ordem são equações cujas derivadas de maior ordem são as de segunda.

A equação diferencial parcial mais básica e que aparece em uma variedade de aplicações é a equação de Laplace, definida como,

$$
\Delta u=0,
$$

em que,

$$
\Delta u=\sum_{i=1}^{n} \frac{\partial^{2} u}{\partial x_{i} \partial x_{j}} .
$$

Para uma maior generalidade, pode-se escrever as equações na forma,

$$
L u=f,
$$

onde $L$ pode ser dado por,

$$
L u=-\sum_{i=1}^{n} \sum_{j=0}^{n} A_{i j}(x) \frac{\partial^{2} u}{\partial x_{i} \partial x_{j}}+\sum_{i=1}^{n} b_{i}(x) \frac{\partial u}{\partial x_{i}}+c(x) u,
$$

$\mathrm{ou}$,

$$
L u=-\sum_{i=1}^{n} \sum_{j=0}^{n} \frac{\partial}{\partial x_{i}}\left(A_{i j}(x) \frac{\partial u}{\partial x_{j}}\right)+\sum_{i=1}^{n} b_{i}(x) \frac{\partial u}{\partial x_{i}}+c(x) u .
$$

A forma (1-2) é dita forma não divergente, e (1-3) é dita forma divergente. Sendo $A_{i j}, b$ e $c$ coeficientes dados, onde comumente se assume $A_{i j}=A_{j i} \mathrm{e}$ notando que caso os coeficientes $A_{i j}$ sejam $C^{1},(1-3)$ pode ser reescrita na forma (1-2).

Equações diferenciais parciais de segunda ordem aparecem tanto em problemas de matemática pura quanto em problemas aplicados . O estudo dos problemas aplicados permite a interpretação dos termos da equação como sendo os termos de difusão, transporte e fonte, respectivamente (4).

Por fim, uma equação diferencial parcial de segunda ordem é dita elíptica se o operador $L$ obedece ao seguinte critério.

Definição 1.1 Diz-se que o operador L é elíptico se existe uma constante 
$\alpha>0$ tal que,

$$
\sum_{i=1}^{n} \sum_{j=1}^{n} A_{i j}(x) \phi_{i} \phi_{j} \geq \alpha|\phi|^{2},
$$

para todo $x \in \Omega$ e para todo $\phi \in \mathbb{R}^{n}$.

Soluções clássicas para problemas envolvendo a equação (1-1) são funções $u \in C^{2}(\Omega) \cap C(\bar{\Omega})$. Porem, pode-se construir uma classe maior de funções que satisfazem (1-1), e outras equações, caso seja exigido menos suavidade de $u$. Para isso deve-se construir um espaço apropriado que leva o nome de espaço de Sobolev (1). 


\section{Espaços de Sobolev}

Neste capítulo será desenvolvida a formulação dos espaços de Sobolev e suas propriedades necessárias ao estudo das soluções fracas para equações elípiticas de segunda ordem.

\section{1}

\section{Definições}

O espaço de Sobolev é construído sobre os espaços $L^{p}$ que são definidos a seguir.

Definição 2.1 O espaço $L^{p}(\Omega)$ é definido como,

$$
L^{p}(\Omega)=\left\{f: \Omega \rightarrow \mathbb{R}, \int_{\Omega}|f|^{p} d x<\infty\right\}
$$

Para a definição dos espaços de Sobolev resta agora definir o conceito de derivada fraca.

Definição 2.2 (Derivada Fraca) Diz-se que $u \in L^{p}(\Omega)$ tem derivada fraca $v_{i} \in L^{p}(\Omega)$ se $\forall \phi \in C_{c}^{\infty}(\Omega)$

$$
\int_{\Omega} u D_{i} \phi d x=-\int_{\Omega} v_{i} \phi d x
$$

Dada a definição de derivada fraca, pode-se ver que se uma derivada forte existe ela é, também, uma derivada fraca.

Teorema 2.3 Se $u \in C^{1}$ e exite $f \in C^{0}$ tal que $\frac{\partial u}{\partial x_{i}}=f$, então

$$
\int_{\Omega} u \frac{\partial \phi}{\partial x_{i}} d x=-\int_{\Omega} f \phi d x
$$

Prova. Se $\frac{\partial u}{\partial x_{i}}=f$, multiplicando por $\phi \in C^{1}(\Omega)$ e integrando por parte tem-se,

$$
\int_{\Omega} u \frac{\partial \phi}{\partial x_{i}} d x=-\int_{\Omega} f \phi d x
$$


Usando a notação $\alpha=\left(\alpha_{1}, \ldots, \alpha_{n}\right)$ onde $\alpha_{i} \in \mathbb{N}, \alpha$ é chamado de multi índice de ordem $|\alpha|=\alpha_{1}+\ldots+\alpha_{n}$ e $D^{\alpha} u=\frac{\partial^{|\alpha|} u}{\partial x_{1}^{\alpha_{1}} \ldots \partial x_{n}^{\alpha_{n}}}$. Deste modo, espaços de Sobolev são os espaços de Banach onde as derivadas fracas pertencem aos espaços $L^{p}$.

Definição 2.4 O espaço de Sobolev $W^{k, p}(\Omega)$ é definido como,

$$
\begin{array}{r}
W^{k, p}(\Omega)=\left\{u \in L^{p}(\Omega)|\forall \alpha,| \alpha \mid \leq k, \exists v_{\alpha} \in L^{p}(\Omega),\right. \\
\left.\int_{\Omega} u D^{\alpha} \phi d x=(-1)^{\alpha} \int_{\Omega} v_{\alpha} \phi d x \forall \phi \in C_{c}^{\infty}(\Omega)\right\}
\end{array}
$$

com $v_{\alpha}=D^{\alpha} u$ derivada fraca e norma,

$$
\|u\|_{W^{k, p}(\Omega)}=\sum_{|\alpha|=0}^{k}\left\|D^{\alpha} u\right\|_{L^{p}(\Omega)} .
$$

A seguir se dão as provas que os espaços de Sobolev são de fato espaços de Banach.

Teorema 2.5 $\|u\|_{W^{k, p}(\Omega)}$ é uma norma.

Prova. Vamos mostrar que,

1. $\|\lambda u\|_{W^{k, p}(\Omega)}=|\lambda|\|u\|_{W^{k, p}(\Omega)}$ para $u \in W^{k, p}(\Omega)$ e $\lambda \in \mathbb{R}$.

$$
\begin{gathered}
\|\lambda u\|_{W^{k, p}(\Omega)}=\sum_{|\alpha|=0}^{k}\left\|D^{\alpha} \lambda u\right\|_{L^{p}(\Omega)}=\sum_{|\alpha|=0}^{k}|\lambda|\left\|D^{\alpha} u\right\|_{L^{p}(\Omega)}, \\
\|\lambda u\|_{W^{k, p}(\Omega)}=|\lambda|\|u\|_{W^{k, p}(\Omega)} .
\end{gathered}
$$

2. $\|u+v\|_{W^{k, p}(\Omega)} \leq\|u\|_{W^{k, p}(\Omega)}+\|v\|_{W^{k, p}(\Omega)} \forall u, v \in W^{k, p}(\Omega)$.

$$
\|u+v\|_{W^{k, p}(\Omega)}=\sum_{|\alpha|=0}^{k}\left\|D^{\alpha} u+D^{\alpha} v\right\|_{L^{p}(\Omega)}
$$

usando a desigualdade de Minkowski,

$$
\begin{gathered}
\|u+v\|_{W^{k, p}(\Omega)} \leq \sum_{|\alpha|=0}^{k}\left\|D^{\alpha} u\right\|_{L^{p}(\Omega)}+\left\|D^{\alpha} v\right\|_{L^{p}(\Omega)}, \\
\|u+v\|_{W^{k, p}(\Omega)} \leq\|u\|_{W^{k, p}(\Omega)}+\|v\|_{W^{k, p}(\Omega)} .
\end{gathered}
$$

Teorema 2.6 O espaço de Sobolev $W^{k, p}(\Omega)$ com norma $\|u\|_{W^{k, p}(\Omega)}$ é um espaço de Banach. 
Prova. Dado 2.5 nos resta mostrar que o espaço de Sobolev é completo. Assim, dado uma sequência de Cauchy $\{u\}_{n=1}^{\infty} \operatorname{em} W^{k, p}(\Omega)$ temos, imediatamente, que $\left\{D^{\alpha} u_{n}\right\}_{n=1}^{\infty}$ em $L^{p}(\Omega)$. Usando o fato que $L^{p}(\Omega)$ é completo podemos afirmar que existe $u_{\alpha}$ tal que $D^{\alpha} u_{n} \rightarrow u_{\alpha}$ em $L^{p}(\Omega)$. Para um $\phi \in C_{c}^{\infty}(\Omega)$ e $|\alpha| \leq k$,

$$
\int_{\Omega} u D^{\alpha} \phi d x=\lim _{n \rightarrow \infty} \int_{\Omega} u_{n} D^{\alpha} \phi d x
$$

Integrando por partes (ver teorema 2 página $712(4)$ ),

$$
\int_{\Omega} u D^{\alpha} \phi d x=\lim _{n \rightarrow \infty}(-1)^{\alpha} \int_{\Omega} D^{\alpha} u_{n} \phi d x=(-1)^{\alpha} \int_{\Omega} u_{\alpha} \phi d x .
$$

Logo, como $u_{n} \rightarrow u$ em $L^{p}(\Omega)$ e existe $u_{\alpha}$ derivada fraca de $u$, temos que $u \in W^{k, p}(\Omega)$.

É possível definir outra norma para os espaços de Sobolev como sendo,

$$
\|u\|_{W^{k, p}}^{(2)}=\left(\|u\|_{p}^{p}+\sum_{|\alpha|=1}^{n}\left\|D^{\alpha} u\right\|^{p}\right)^{\frac{1}{p}}
$$

e usando o corolário do teorema das aplicações abertas (ver corolário $2.8(2)$ ) garante a equivalência das normas, uma vez que

$$
\|u\|_{W^{k, p}}^{(2)} \leq\|u\|_{W^{k, p}}
$$

\section{2}

\section{Propriedades dos Espaços de Sobolev}

Uma vez que se tenha demonstrado que os espaços de Sobolev são espaços de Banach, as seguintes propriedades serão provadas.

Teorema 2.7 Se $\rho \in L^{p}(\Omega)$ com suporte compacto e $u \in W^{1, p}(\Omega)$ com $1 \leq p<\infty$, então, $\rho \star u \in W^{1, p}(\Omega) e$

$$
\frac{\partial}{\partial x_{i}}(\rho \star u)=\rho \star \frac{\partial u}{\partial x_{i}} .
$$

Prova. Se $\rho \in L^{1}(\Omega)$ e $u \in W^{1, p}(\Omega)$ então $\rho \star u \in L^{p}(\Omega)$. Da teoria de espaços $L^{p}$ sabe-se que se $f \in C_{c}^{k}(\Omega)$ e $g \in L^{1}(\Omega)$, então $f \star g \in C^{k}(\Omega)$ e ainda, $D^{\alpha}(f \star g)=\left(D^{\alpha} f\right) \star g$ e dado $f \in L^{1}(\Omega), g \in L^{p}(\Omega), h \in L^{p^{\prime}}(\Omega) \operatorname{com} \hat{f}$ definido como $\hat{f}=f(-x)$ tem-se $\int_{\Omega}(f \star g) h=\int_{\Omega} g(\hat{f} \star h)$ (ver teorema $4.15 \mathrm{e}$ proposições 4.16 e $4.20(2))$. Com esses fatos, tem-se,

$$
\int_{\Omega}(\rho \star u) \frac{\partial \phi}{\partial x_{i}} d x=\int_{\Omega} u\left(\hat{\rho} \star \frac{\partial \phi}{\partial x_{i}}\right) d x=\int_{\Omega} u \frac{\partial}{\partial x_{i}}(\hat{\rho} \star \phi) d x,
$$


integrando por partes,

$$
\int_{\Omega} u \frac{\partial}{\partial x_{i}}(\hat{\rho} \star \phi) d x=-\int_{\Omega} \frac{\partial u}{\partial x_{i}}(\hat{\rho} \star \phi) d x=\int_{\Omega}\left(\rho \star \frac{\partial u}{\partial x_{i}}\right) \phi d x,
$$

chegando, assim, em,

$$
\int_{\Omega}(\rho \star u) \frac{\partial \phi}{\partial x_{i}} d x=-\int_{\Omega}\left(\rho \star \frac{\partial u}{\partial x_{i}}\right) \phi d x
$$

concluindo a prova.

Definição 2.8 (Função de Corte) Seja $\xi \in C_{c}^{\infty}\left(\mathbb{R}^{n}\right) \operatorname{com} 0 \leq \xi \leq 1$ e

$$
\xi(x)= \begin{cases}1 & |x| \leq 1 \\ 0 & |x|>2\end{cases}
$$

e defina $\xi_{n}(x)=\xi\left(\frac{x}{n}\right)$ com $n=1,2, \ldots$

Teorema 2.9 (Friedrichs) Seja $u \in W^{1, p}(\Omega)$ com $1 \leq p<\infty$. Então existe uma sequência $\left\{u_{n}\right\}$ em $C_{c}^{\infty}\left(\mathbb{R}^{n}\right)$ tal que,

$$
\left.u_{n}\right|_{\Omega} \rightarrow u e m L^{p}(\Omega),
$$

$e$

$$
\left.\left.D u_{n}\right|_{\omega} \rightarrow D u\right|_{\omega}, \quad L^{p}(\omega) \forall \omega \subset \subset \Omega
$$

Prova. Seja,

$$
\bar{u}(x)= \begin{cases}u(x) & x \in \Omega \\ 0 & x \in \Omega^{c}\end{cases}
$$

definindo $v_{n}=\rho_{n} \star u$, onde $\rho_{n}$ é uma sequência de mollifiers (ver página 108 (2)), sabe-se que $v_{n} \in C_{c}^{\infty}\left(\mathbb{R}^{n}\right)$ e $v_{n} \rightarrow \bar{u}$ em $L^{p}(\Omega)$. Seja $\omega \subset \subset \Omega$ e $\alpha \in C_{c}^{1}(\Omega)$ tal que $0 \leq \alpha \leq 1$ e $\alpha=1$ na vizinhança de $\omega$. Como, para $n$ suficientemente grande,

$$
\begin{array}{r}
\operatorname{supp}\left(\rho_{n} \star \overline{\alpha u}-\rho_{n} \star \bar{u}\right)=\operatorname{supp}\left(\rho_{n} \star(1-\bar{\alpha}) \bar{u}\right), \\
\subset \overline{\operatorname{supp} \rho_{n}+\operatorname{supp}\left(\rho_{n} \star(1-\bar{\alpha}) \bar{u}\right)}, \\
\subset \overline{\left(B\left(0, \frac{1}{n}\right)+\operatorname{supp}(1-\bar{\alpha}),\right.} \\
\subset \omega^{c},
\end{array}
$$

tem-se que, 


$$
\rho_{n} \star(\overline{\alpha u})=\rho_{n} \star \bar{u} \text { em } \omega .
$$

Segue, uma vez que,

$$
\int_{\mathbb{R}^{n}} \overline{\alpha u} \frac{\partial \phi}{\partial x_{i}} d x=\int_{\omega} \alpha u \frac{\partial \phi}{\partial x_{i}}=-\int_{\omega} \frac{\partial}{\partial x_{i}}(\alpha u) \phi d x=-\int_{\omega}\left(\alpha \frac{\partial u}{\partial x_{i}}+\frac{\alpha}{\partial x_{i}} u\right) \phi d x
$$

e que,

$$
\begin{gathered}
\int_{\omega}\left(\alpha \frac{\partial u}{\partial x_{i}}+\frac{\partial \alpha}{\partial x_{i}} u\right) \phi d x=\int_{\mathbb{R}^{n}}\left(\overline{\alpha \frac{\partial u}{\partial x_{i}}+\frac{\partial \alpha}{\partial x_{i}} u}\right) \phi d x, \\
\frac{\partial}{\partial x_{i}}\left(\rho_{n} \star \overline{\alpha u}\right) \rightarrow \overline{\frac{\partial u}{\partial x_{i}}+\frac{\partial \alpha}{\partial x_{i}} u} \text { em } L^{p}\left(\mathbb{R}^{n}\right),
\end{gathered}
$$

e tem-se que,

$$
\frac{\partial}{\partial x_{i}}\left(\rho_{n} \star \overline{\alpha u}\right) \rightarrow \frac{\partial u}{\partial x_{i}} \text { em } L^{p}(\omega)
$$

devido a 2-4

$$
\frac{\partial}{\partial x_{i}}\left(\rho_{n} \star \bar{u}\right) \rightarrow \frac{\partial u}{\partial x_{i}} \text { em } L^{p}(\omega)
$$

Multiplicando $v_{n}$ pelas funções de corte $\xi_{n}$ tem-se $u_{n}=\xi_{n} v_{n} \in C_{c}^{\infty}\left(\mathbb{R}^{n}\right)$ tal que $u_{n} \rightarrow u$ em $L^{p}(\Omega)$ e $D u_{n} \rightarrow D u$ em $L^{p}(\omega)$.

Teorema 2.10 Seja $G \in C^{1}(\mathbb{R})$ tal que $G(0)=0$ e $\left|G^{\prime}(t)\right| \leq M \forall t \in \mathbb{R}^{n}$ e para uma constante $M$. Se $u \in W^{1, p}(\Omega)$ e $1 \leq p \leq \infty$ então,

$$
G \circ u \in W^{1, p}(\Omega)
$$

$e$,

$$
\frac{\partial}{\partial x_{i}}(G \circ u)=\left(G^{\prime} \circ u\right) \frac{\partial u}{\partial x_{i}}
$$

para $i=1,2, \ldots, n$.

Prova. Dos pressupostos temos $|G(t)-G(0)| \leq M|t|$, logo $|G(u)| \leq M|u|$, assim é imediato que $G \circ u \in L^{p}(\Omega)$ e $\left(G^{\prime} \circ u\right) \frac{\partial u}{\partial x_{i}} \in L^{p}(\Omega)$, logo $G \circ u \in W^{1, p}(\Omega)$. Agora resta mostrar que,

$$
\int_{\Omega}(G \circ u) \frac{\partial \phi}{\partial x_{i}} d x=-\int_{\Omega}\left(G^{\prime} \circ u\right) \frac{\partial u}{\partial x_{i}} \phi d x
$$

para todo $\phi \in C_{c}^{1}(\Omega)$. Usando (2.9) tem-se o resultado desejado.

O seguinte teorema trata da densidade dos espaços $C^{\infty}$ nos espaços $W^{k, p}$.

Teorema 2.11 (Lema de Mayers-Serrin) $C^{\infty}(\Omega) \cap W^{k, p}(\Omega)$ é denso em $W^{k, p}(\Omega)$.

Prova. Seja $u \in W^{k, p}(\Omega)$ e defina $\Omega_{i}$ como,

$$
\Omega_{i}=\{x \in \Omega,|x| \leq i, \operatorname{dist}(x, \partial \Omega)>1 / i\}
$$


para $i=1,2, \ldots$ Definindo $\Omega_{-1}=\Omega_{0}=\emptyset$ e $A_{i}=\Omega_{i+1} \backslash \Omega_{i-1}$ tem-se que $\cup A_{i} \supset \Omega$. Agora, seja $\left\{\psi_{i}\right\}$ a partição de unidade (ver seção $6.9(5)$ ) sujeita a $\left\{A_{i}\right\}$, sejam $\rho_{n} \in C^{\infty}(\Omega)$ os mollifiers, dado $\epsilon>0$ tem-se que,

$$
\left\|\rho_{n} \star \psi_{n} u-\psi_{n} u\right\|_{W^{k, p}} \leq \epsilon .
$$

Definindo $v=\sum_{i=1}^{\infty} \rho_{n} \star \psi_{n} u$ e do fato que $\sum_{n=1}^{\infty} \psi_{n}=1$ pode-se escrever $u=\sum_{u=1}^{\infty} \psi_{n} u$, assim,

$$
\|u-v\|_{W^{k, p}}=\left\|\sum_{n=1}^{\infty} \psi_{n} u-\rho_{n} \star \psi_{n} u\right\|_{W^{k, p}} \leq \sum_{n=1}^{\infty}\left\|\psi_{n} u-\rho_{n} \star \psi_{n} u\right\|_{W^{k, p}},
$$

com (2-5) tem-se que para todo $\epsilon>0$ existe $v \in C^{\infty}(\Omega)$ tal que,

$$
\|u-v\|_{W^{k, p}} \leq \epsilon
$$

\section{3}

\section{Desigualdades de Sobolev}

Desenvolvida as propriedades básicas dos espaços de Sobolev, pode-se perguntar sobre possíveis inclusões em outros espaços. Essas inclusões serão uteis, uma vez que no estudo das equações diferenciais parciais se define a dimensão e o índice $p$, para saber em qual espaço as soluções estarão incluídas.

\subsection{1}

\section{Gagliardo-Nirenberg-Sobolev}

Teorema 2.12 (Desigualdade de Gagliardo-Nirenberg-Sobolev) Dado $1 \leq p<$ n e $u \in C_{c}^{1}\left(\mathbb{R}^{n}\right)$ existe $C=C(n, p)$ tal que,

$$
\|u\|_{p^{*}} \leq C\|D u\|_{p}
$$

onde $p^{*}$ é dado por,

$$
\frac{1}{p^{*}}=\frac{1}{p}-\frac{1}{n}
$$

Prova. Vamos considerar primeiro o caso $p=1$ para, a partir dele, chegar no resultado geral. Pelo teorema fundamental do cálculo e pelo fato de $u$ ter suporte compacto,

$$
u(x)=\int_{-\infty}^{x_{i}} D_{i} u d y_{i}
$$


tomando o modulo e usando a desigualdade de Jensen,

$$
\begin{gathered}
|u(x)|=\left|\int_{-\infty}^{x_{i}} D_{i} u d y_{i}\right| \\
|u(x)| \leq \int_{-\infty}^{x_{i}}\left|D_{i} u\right| d y_{i} \leq \int_{-\infty}^{\infty}\left|D_{i} u\right| d y_{i} \leq \int_{-\infty}^{\infty}|D u| d y_{i} .
\end{gathered}
$$

Tomando o produto para cada $i=1, \ldots, n$ e a $n-1$ ésima raiz,

$$
|u(x)|^{\frac{n}{n-1}} \leq \prod_{i=1}^{n}\left(\int_{-\infty}^{\infty}|D u| d y_{i}\right)^{\frac{1}{n-1}}
$$

integrando em relação a $x_{k}, \operatorname{com} k=1, \ldots, n$, a integral com respeito a $y_{k}$ não dependerá de $x_{k}$ ficando assim,

$$
\int_{-\infty}^{\infty}|u(x)|^{\frac{n}{n-1}} d x_{k} \leq\left(\int_{-\infty}^{\infty}|D u| d y_{k}\right)^{\frac{1}{n-1}} \prod_{i=1, i \neq k}^{n}\left(\int_{-\infty}^{\infty}|D u| d y_{i}\right)^{\frac{1}{n-1}}
$$

definindo $f_{i}=\left(\int_{-\infty}^{\infty}|D u| d y_{i}\right)^{\frac{1}{n-1}}$

$$
\int_{-\infty}^{\infty}|u(x)|^{\frac{n}{n-1}} d x_{k} \leq\left(\int_{-\infty}^{\infty}|D u| d y_{k}\right)^{\frac{1}{n-1}} \int_{-\infty}^{\infty} f_{1} \ldots f_{k-1} f_{k+1} \ldots f_{n} d x_{k}
$$

aplicando a desigualdade de Hölder, com $p_{m}=n-1$ de modo que $\sum_{m}^{n-1} \frac{1}{p_{m}}=1$, o resultado será,

$$
\begin{gathered}
\int_{-\infty}^{\infty}|u(x)|^{\frac{n}{n-1}} d x_{k} \leq\left(\int_{-\infty}^{\infty}|D u| d y_{k}\right)^{\frac{1}{n-1}} \prod_{i=1, i \neq k}^{n}\left\|f_{i}\right\|_{p_{m}}, \\
\int_{-\infty}^{\infty}|u(x)|^{\frac{n}{n-1}} d x_{k} \leq\left(\int_{-\infty}^{\infty}|D u| d y_{k}\right)^{\frac{1}{n-1}} \prod_{i=1 \neq i=k}^{n}\left[\int_{-\infty}^{\infty}\left[\left(\int_{-\infty}^{\infty}|D u| d y_{i}\right)^{\frac{1}{n-1}}\right]^{n-1} d x_{k}\right]^{\frac{1}{n-1}} \\
\int_{-\infty}^{\infty}|u(x)|^{\frac{n}{n-1}} d x_{k} \leq\left(\int_{-\infty}^{\infty}|D u| d y_{k}\right)^{\frac{1}{n-1}} \prod_{i=1 \neq i=k}^{n}\left(\int_{-\infty}^{\infty} \int_{-\infty}^{\infty}|D u| d y_{i} d x_{k}\right)^{\frac{1}{n-1}}
\end{gathered}
$$

deste modo, integrando em respeito a $x_{1}$ resultará em,

$$
\int_{-\infty}^{\infty}|u(x)|^{\frac{n}{n-1}} d x_{1} \leq\left(\int_{-\infty}^{\infty}|D u| d y_{k}\right)^{\frac{1}{n-1}} \prod_{i=2}^{n}\left(\int_{-\infty}^{\infty} \int_{-\infty}^{\infty}|D u| d y_{i} d x_{1}\right)^{\frac{1}{n-1}} .
$$

Repetindo o processo para $x_{2}, \ldots, x_{n}$,

$$
\int_{-\infty}^{\infty}|u(x)|^{\frac{n}{n-1}} d x \leq \prod_{i=1}^{n}\left(\int_{-\infty}^{\infty} \ldots \int_{-\infty}^{\infty}|D u| d x_{1} \ldots d y_{i} \ldots d x_{n}\right)^{\frac{1}{n-1}}=\left(\int_{\mathbb{R}^{n}}|D u| d x\right)^{\frac{n}{n-1}}
$$


tomando a $\frac{n-1}{n}$ raiz,

$$
\begin{gathered}
\left(\int_{-\infty}^{\infty}|u(x)|^{\frac{n}{n-1}} d x\right)^{\frac{n-1}{n}} \leq\left(\int_{\mathbb{R}^{n}}|D u| d x\right) \\
\|u\|_{1^{*}} \leq\|D u\|_{1} .
\end{gathered}
$$

Onde $1^{*}=\frac{n}{n-1}$, provando para o caso $p=1$. Para o caso $1<p<n$, tome a potência $\gamma>1$ de $|u|$,

$$
\left\||u|^{\gamma}\right\|_{\frac{n}{n-1}} \leq\left.\left.\int_{\mathbb{R}^{n}}|D| u\right|^{\gamma}\left|d x=\gamma \int_{\mathbb{R}^{n}}\right| u\right|^{\gamma-1}|D u| d x
$$

usando a desigualdade de Hölder,

$$
\left\||u|^{\gamma}\right\|_{\frac{n}{n-1}} \leq\left.\gamma\|\| u\right|^{\gamma-1}\left\|_{q}\right\| D u \|_{p}
$$

pode-se escolher $\gamma$ de modo que $\frac{\gamma n}{n-1}=\frac{(\gamma-1) p}{p-1}=\frac{n p}{n-p}$ e $\gamma=\frac{p(n-1)}{n-p}$, notando também que $\frac{1}{q}+\frac{1}{p}=1 \Rightarrow q=\frac{p}{p-1}$. O resultado será,

$$
\begin{gathered}
\left(\int_{\mathbb{R}^{n}}\left(|u|^{\gamma}\right)^{\frac{n}{n-1}} d x\right)^{\frac{n-1}{n}} \leq \gamma\left(\int_{\mathbb{R}^{n}}\left(|u|^{\gamma-1}\right)^{q} d x\right)^{\frac{1}{q}}\|D u\|_{p}, \\
\left(\int_{\mathbb{R}^{n}}|u|^{\gamma \frac{n}{n-1}} d x\right)^{\frac{n-1}{n}} \leq \gamma\left(\int_{\mathbb{R}^{n}}|u|^{(\gamma-1) \frac{p}{p-1}} d x\right)^{\frac{p-1}{p}}\|D u\|_{p}, \\
\left(\int_{\mathbb{R}^{n}}|u|^{\frac{n p}{n-p}} d x\right)^{\frac{n-1}{n}} \leq \gamma\left(\int_{\mathbb{R}^{n}}|u|^{\frac{n p}{n-p}} d x\right)^{\frac{p-1}{p}}\|D u\|_{p},
\end{gathered}
$$

sabendo que $\frac{n-1}{n}-\frac{p-1}{p}=\frac{n-p}{n p}$

$$
\left(\int_{\mathbb{R}^{n}}|u|^{\frac{n p}{n-p}} d x\right)^{\frac{n-p}{n p}} \leq \frac{p(n-1)}{n-p}\|D u\|_{p}
$$

concluindo a prova.

Corolário 2.13 $W^{1, p}(\Omega) \subset L^{p^{*}}(\Omega)$

Prova. Uma vez que

$$
\|u\|_{p *} \leq C\|u\|_{p}
$$

tem-se

$$
\|u\|_{p *} \leq C\|u\|_{W^{1, p}}
$$

\subsection{2}

$n=p$

Teorema 2.14 $W^{1, p}(\Omega) \subset L^{q} \forall q \in[n, \infty)$ 
Prova. dado $u \in C_{c}^{1}$, de (2-6) tem-se,

$$
\left\|u^{\gamma}\right\|_{q} \leq n\left\|u^{\gamma-1}\right\|_{q}\|D u\|_{n} \text {,fazendo } p=\gamma=n
$$

usando a desigualdade de Young,

$$
\left\|u^{n}\right\|_{q} \leq C\left(\left\|u^{n-1}\right\|_{q}^{q}+\|D u\|_{n}^{n}\right)
$$

, notando que a desigualdade a cima é a mesma que,

$$
\left(\int_{\mathbb{R}^{n}}|u|^{n q} d x\right)^{\frac{1}{q}} \leq C\left(\int_{\mathbb{R}^{n}}|u|^{(n-1) q} d x+\int_{\mathbb{R}^{n}}|D u|^{n} d x\right)
$$

observado o fato $q=\frac{p}{p-1}$ resulta em $(n-1) q=n$ e $n q=\frac{n^{2}}{n-1}$,

$$
\begin{gathered}
\left(\int_{\mathbb{R}^{n}}|u|^{\frac{n^{2}}{n-1}} d x\right)^{\frac{n-1}{n}} \leq C\left(\int_{\mathbb{R}^{n}}|u|^{n} d x+\int_{\mathbb{R}^{n}}|D u|^{n} d x\right)=C\left(\|u\|_{n}^{n}+\|D u\|_{n}^{n}\right), \\
\leq C\left(\|u\|_{n}+\|D u\|_{n}\right)^{n},
\end{gathered}
$$

tomando a n-ésima raiz,

$$
\|u\|_{\frac{n^{2}}{n-1}} \leq C\|u\|_{W^{1, p}}
$$

Usando a desigualdade de interpolação com $n \leq q \leq \frac{n^{2}}{n-1}$ e $\alpha=0$ tem-se que,

$$
\|u\|_{q} \leq C\|u\|_{W^{1, p}}
$$

\subsection{3}

\section{Morrey}

Teorema 2.15 (Morrey) $W^{1, p}\left(\mathbb{R}^{n}\right) \subset L^{\infty}\left(\mathbb{R}^{n}\right)$

Prova. Seja $u \in C_{c}^{1}\left(\mathbb{R}^{n}\right)$ e $Q$ um cubo aberto de lado $l$ com as arestas paralelas aos eixos tal que $0 \in Q$, tomando $x \in Q$ o teorema fundamental do calculo fornece,

$$
u(x)-u(0)=\int_{0}^{1} \frac{d}{d t} u(t x) d t \text { para } t \in(0,1),
$$

integrando sobre o cubo $Q$, temos,

$$
\frac{1}{|Q|} \int_{Q} u(x) d x-u(0) \frac{1}{|Q|} \int_{Q} d x=\frac{1}{|Q|} \int_{Q} \int_{0}^{1} \frac{d}{d t} u(t x) d t d x
$$


definindo $\bar{u}=\frac{1}{|Q|} \int_{Q} u(x) d x$

$$
\bar{u}-u(0)=\frac{1}{|Q|} \int_{Q} \int_{0}^{1} \frac{d}{d t} u(t x) d t d x=\frac{1}{|Q|} \int_{Q} \int_{0}^{1} \sum_{i=0}^{n} \frac{\partial}{\partial x_{i}} u\left(t x_{i}\right) x_{i} d t d x
$$

tomando o modulo e usando a desigualdade de Jensen,

$$
|\bar{u}-u(0)| \leq \frac{1}{|Q|} \int_{Q} \int_{0}^{1}\left|\sum_{i=0}^{n} \frac{\partial}{\partial x_{i}} u\left(t x_{i}\right) x_{i}\right| d t d x
$$

como $x \in Q$ e $\left|x_{i}\right| \leq l \forall i \leq n$,

$$
|\bar{u}-u(0)| \leq \frac{l}{|Q|} \int_{Q} \int_{0}^{1}\left|\sum_{i=0}^{n} \frac{\partial}{\partial x_{i}} u\left(t x_{i}\right)\right| d t d x,
$$

fazendo a mudança derivável $x t=y$ e observando que $t Q \subset Q$,

$$
|\bar{u}-u(0)| \leq \frac{l}{|Q|} \int_{t Q} \int_{0}^{1}\left|\sum_{i=0}^{n} \frac{\partial}{\partial x_{i}} u\left(y_{i}\right)\right| d t \frac{d y}{t^{n}},
$$

sabendo que $|Q|=l^{n}$,

$$
|\bar{u}-u(0)| \leq \frac{1}{l^{n-1}} \int_{t Q} \int_{0}^{1}\left|\sum_{i=0}^{n} \frac{\partial}{\partial x_{i}} u\left(y_{i}\right)\right| d t \frac{d y}{t^{n}},
$$

agora, observando,

$$
\int_{t Q}\left|\sum_{i=0}^{n} \frac{\partial}{\partial x_{i}} u\left(y_{i}\right)\right| d y=\|D u\|_{L^{1}(t Q)}
$$

usando a desigualdade de Hölder,

$$
\|D u\|_{L^{1}(t Q)} \leq\|D u\|_{L^{p}(t Q)}\|1\|\left\|_{L^{p^{\prime}}(t Q)}=\right\| D u \|_{L^{p}(t Q)}|t Q|^{\frac{1}{p^{\prime}}}
$$

voltando para (2-7),

$$
|\bar{u}-u(0)| \leq \frac{l^{n / p^{\prime}}}{l^{n-1}}|| D u \|_{L^{p}(Q)} \int_{0}^{1} \frac{t^{n / p^{\prime}}}{t^{n}} d t,
$$

notando que $\frac{n}{p^{\prime}}-(n-1)=1-\frac{n}{p}$ e $\frac{n}{p^{\prime}-n}=-\frac{n}{p}$ temos,

$$
|\bar{u}-u(0)| \leq l^{1-\frac{n}{p}}|| D u \|_{L^{p}(Q)} \int_{0}^{1} t^{-\frac{n}{p}} d t
$$

que é equivalente a,

$$
|\bar{u}-u(0)| \leq \frac{l^{1-\frac{n}{p}}}{1-\frac{n}{p}}\|D u\|_{L^{p}(Q)},
$$


transladando para $x \in Q$,

$$
\begin{gathered}
|\bar{u}-u(x)| \leq \frac{l^{1-\frac{n}{p}}}{1-\frac{n}{p}}\|D u\|_{L^{p}(Q)} \\
|u|-|\bar{u}| \leq|\bar{u}-u(x)| \leq C|| D u \|_{L^{p}(Q)} \\
|u| \leq|\bar{u}|+C|| D u \|_{L^{p}(Q)}
\end{gathered}
$$

agora, notando que por Hölder,

$$
\frac{1}{|Q|} \int_{Q}|u| d x \leq\|u\|_{L^{p}(Q)} \frac{|Q|^{1 / p^{\prime}}}{|Q|} \leq C\|u\|_{L^{p}(Q)}
$$

assim,

$$
|u| \leq C|| u\left\|_{L^{p}(Q)}+\right\| D u \|_{L^{p}(Q)},
$$

chegando em,

$$
|u| \leq C\|u\|_{W^{1, p}\left(\mathbb{R}^{n}\right)}
$$

uma vez que $u$ é qualquer, tem-se o resultado.

Usando (2-8) pode-se, também, obter uma estimativa para funções em $W^{1, p}\left(\mathbb{R}^{n}\right)$.

Teorema 2.16 Para todo $u \in W^{1, p}\left(\mathbb{R}^{n}\right)$,

$$
|u(x)-u(y)| \leq C|x-y|^{1-\frac{n}{p}}|| D u \|_{L^{p}\left(\mathbb{R}^{n}\right)},
$$

para $x, y \in \mathbb{R}^{n}$,

Prova. Tendo $u \in C_{c}^{1}\left(\mathbb{R}^{n}\right), x, y \in \mathbb{R}^{n}$ e (2-8), chega-se a,

$$
|-\bar{u}+u(x)|+|\bar{u}-u(y)| \geq|-\bar{u}+u(x)+\bar{u}-u(y)|=|u(x)-u(y)|,
$$

de modo que,

$$
|u(x)-u(y)| \leq 2 \frac{l^{1-\frac{n}{p}}}{1-\frac{n}{p}}|| D u \|_{L^{p}(Q)},
$$

para um $Q$ tal que $x, y$ que estejam em $Q$ e escolhendo $l$ de maneira que $l=C|x-y|<\infty$ tem-se o resultado desejado.

$$
|u(x)-u(y)| \leq C|x-y|^{1-\frac{n}{p}}|| D u \|_{L^{p}\left(\mathbb{R}^{n}\right)}
$$




\section{4}

\section{Espaço $W_{0}^{1, p}$}

Um espaço que será útil ao estudo das soluções fracas para equações diferenciais parciais elípticas é o seguinte.

Definição 2.17 Espaço $W_{0}^{1, p}(\Omega)$ que é definido como o fecho de $C_{c}^{1}(\Omega)$ em $W^{1, p}(\Omega)$.

Para caracterizar funções em $W_{0}^{1, p}$ a proposição a seguir será necessária.

Proposição 2.18 Se $u \in W^{1, p}(\Omega)$ com $1 \leq p<\infty$ e suporte compacto, então $u \in W_{0}^{1, p}(\Omega)$.

Prova. A prova dessa proposição é um resultado do teorema (2.9), uma vez que este garante a existência de $u_{n}$ em $C_{c}^{\infty}\left(\mathbb{R}^{n}\right)$ tal que $u_{n} \rightarrow u$ em $L^{p}(\Omega)$ e $D u_{n} \rightarrow D u$ em $L^{p}(\omega)$. Assim, dado um $\omega$ tal que suppu $\subset \omega \subset \subset \Omega$ e $\alpha$ tal que $\alpha=1$ no suporte de $\mathrm{u}$ de tal modo que $\alpha u=u$, tem-se que $\alpha u_{n} \rightarrow \alpha u$ em $W^{1, p}(\Omega)$, logo $\alpha u \in W_{0}^{1, p}(\Omega)$ e imediatamente $u \in W_{0}^{1, p}(\Omega)$.

Agora, para caracterizar o comportamento de funções no espaço resta dar-se a definição de duas classe de objetos, usando como notação,

$$
\begin{aligned}
& -\mathbb{R}_{+}^{n}=\left\{x=\left(x^{\prime}, x_{N}\right) ; x_{N}>0\right\} \\
& -Q=\left\{x=\left(x^{\prime}, x_{N}\right) ;\left|x^{\prime}\right|<1,\left|x_{N}\right|<1\right\} \\
& -Q_{+}=Q \cap \mathbb{R}_{+}^{n} \\
& -Q_{0}=\left\{x=\left(x^{\prime}, 0\right) ;\left|x^{\prime}\right|<1\right\} .
\end{aligned}
$$

Definição 2.19 $O$ conjunto $\Omega$ é dito de classe $C^{1}$ se para todo $x \in \partial \Omega$ existe uma vizinhança $U \subset \mathbb{R}^{n}$ e uma bijeção $\psi: Q \rightarrow U$ tal que,

$$
\begin{aligned}
& -\psi \in C^{1}(\bar{Q}) \\
& -\psi^{-1} \in C^{1}(\bar{U}) \\
& -\psi\left(Q_{+}\right)=U \cap Q \\
& -\psi\left(Q_{0}\right)=U \cap \partial \Omega
\end{aligned}
$$

$\psi$ é dito um mapa local.

Teorema 2.20 Suponha que $\Omega$ seja de classe $C^{1}$ e $u \in W^{1, p}(\Omega) \cap C(\bar{\Omega})$ com $1 \leq p<\infty$. Então, $u=0$ em $\partial \Omega$ se, e somente se, $u \in W_{0}^{1, p}(\Omega)$. 
Prova. $(\Rightarrow)$ suponha que o suporte de u é limitado e definindo $G \in C^{1}\left(\mathbb{R}^{n}\right)$ tal que $|G(t)| \leq t \quad \forall t \in \mathbb{R}$ e

$$
G(t)= \begin{cases}0 & |t| \leq 1 \\ t & |t| \geq 2\end{cases}
$$

Então, sabe-se que $u_{n}=\frac{1}{n} G(n u) \in W^{1, p}(\Omega)$ e pelo teorema da convergência dominada, $u_{n} \rightarrow u$ em $W^{1, p}(\Omega)$. Tem-se também, que,

$$
\text { supp } u_{n} \subset\{x \in \Omega ;|u(x)| \geq 1 / n\}
$$

assim, supp $u$ é compacto e pela proposição anterior tem-se que $u \in W_{0}^{1, p}(\Omega)$, caso suppu não seja limitado pode-se tomar a sequência $\xi_{n} u$ onde $\xi_{n}$ é a função de corte como definida em (2.8).

$(\Leftarrow)$ Fazendo uso dos mapas locais, pode-se trabalhar no problema onde $u \in W_{0}^{1, p}\left(Q_{+}\right) \cap C\left(\overline{Q_{+}}\right)$e provar que $u=0$ em $Q_{+}$. Assim, tomando $u_{n}$ uma sequência em $C_{c}^{1}\left(Q_{+}\right)$tal que $u_{n} \rightarrow u$ em $W^{1, p}\left(Q_{+}\right)$. tem-se que para $\left(x^{\prime}, x_{N}\right) \in Q_{+}$,

$$
u_{n}\left(x^{\prime}, x_{N}\right)=\int_{0}^{x_{N}} \frac{\partial u_{n}}{\partial x_{N}}\left(x^{\prime}, t\right) d t
$$

assim, pela desigualdade de Jensen,

$$
\left|u_{n}\left(x^{\prime}, x_{N}\right)\right| \leq \int_{0}^{x_{N}}\left|\frac{\partial u_{n}}{\partial x_{N}}\left(x^{\prime}, t\right)\right| d t .
$$

Para $0<r<1$,

$$
\frac{1}{r} \int_{\left|x^{\prime}\right|<1} \int_{0}^{r}\left|u_{n}\left(x^{\prime}, x_{N}\right)\right| d x^{\prime} d x_{N} \leq \int_{\left|x^{\prime}\right|<1} \int_{0}^{r}\left|\frac{\partial u_{n}}{\partial x_{N}}\left(x^{\prime}, t\right)\right| d t,
$$

e para $n$ suficientemente grande,

$$
\int_{\left|x^{\prime}\right|<1} \int_{0}^{r}\left|u\left(x^{\prime}, x_{N}\right)\right| d x^{\prime} d x_{N} \leq r \int_{\left|x^{\prime}\right|<1} \int_{0}^{r}\left|\frac{\partial u}{\partial x_{N}}\left(x^{\prime}, t\right)\right| d t
$$

fazendo $r \rightarrow 0$ tem-se,

$$
\int_{\left|x^{\prime}\right|<1}\left|u\left(x^{\prime}, x_{N}\right)\right| d x^{\prime}=0
$$

$\log \mathrm{o}, u=0$ em $Q_{0}$.

\section{5}

\section{Espaços de Sobolev e Hilbert}

Uma vez desenvolvida a teoria de espaços de Sobolev é possível identificar o espaço de Sobolev $W^{1,2}(\Omega)$ com o espaço de Hilbert $H^{1}(\Omega)$. 
Teorema 2.21 A função (.,.): $W^{1,2} \times W^{1,2} \rightarrow \mathbb{R}$ definido como

$$
(u, v)=\int_{\Omega} u v+D u D v d x
$$

é um produto interno.

Prova. Sejam $u, v, w \in W^{1,2}(\Omega)$, tem-se,

$$
\begin{gathered}
(a u+b v, w)=\int_{\Omega}(a u+b v)+D(a u+b v) D w d x \\
a \int_{\Omega} u w+D u D w d x+b \int_{\Omega} v w+D v D w d x=a(u, w)+b(v, w),
\end{gathered}
$$

e $(u, u) \geq 0$.

De tal forma, para $(u, u)$ com $u \in W^{1,2}(\Omega)$ tem-se,

$$
(u, u)=\int_{\Omega}|u|^{2}+|D u|^{2} d x .
$$

Assim, segue que,

Corolário 2.22 Se $u \in W^{1,2}(\Omega)$ e tem-se (2.21) então,

$$
(u, u)^{\frac{1}{2}}=\|u\|_{W^{1,2}} .
$$

Assim, escrevemos $H^{1}(\Omega)=W^{1,2}(\Omega)$. Agora, pode-se mostrar a desigualdade de Poincaré,

Teorema 2.23 (Poincaré) Seja $\Omega$ limitado, e $u \in H_{0}^{1}(\Omega)$. Então,

$$
\|u\|_{2} \leq C\|D u\|_{2}
$$

Prova. Uma vez que $C^{\infty}$ é denso em $H_{0}^{1}(\Omega)$ basta provar o teorema para $u \in C^{\infty}(\Omega)$. Seja $x^{\prime}=\left(x_{1}, \ldots, x_{n-1}\right)$,

$$
u\left(x^{\prime}, x_{n}\right)=\int_{-\infty}^{x_{n}} \frac{\partial u}{\partial x_{n}}\left(x^{\prime}, t\right) d t
$$

usando a desigualdade de Jensen,

$$
\begin{aligned}
\left|u\left(x^{\prime}, x_{n}\right)\right|^{2} & \leq \int_{-\infty}^{\infty}\left|\frac{\partial u}{\partial x_{n}}\left(x^{\prime}, t\right)\right|^{2} d t \\
\left|u\left(x^{\prime}, x_{n}\right)\right|^{2} & \leq \int_{\infty}^{\infty}\left|D u\left(x^{\prime}, t\right)\right|^{2} d t .
\end{aligned}
$$


Integrando com respeito a $x_{n}$ tem-se,

$$
\int_{\infty}^{\infty}\left|u\left(x^{\prime}, x_{n}\right)\right|^{2} d x_{n} \leq C \int_{\infty}^{\infty}\left|D u\left(x^{\prime}, t\right)\right|^{2} d t
$$

integrando nas demais variáveis, tem-se o resultado. 


\section{Soluções Fracas}

Tendo o espaço de Sobolev e desenvolvido suas suas propriedades básicas este capítulo tratará do desenvolvimento do teorema de Lax-Milgram. Este teorema permitirá o uso da análise funcional para o estudo da existência e unicidade de soluções fracas.

\section{1}

\section{O Teorema de Lax-Milgram}

As formas bilineares são fundamentais para o teorema de Lax-Milgram e são definidas a seguir.

Definição 3.1 Uma forma bilinear a: $H \times H \rightarrow \mathbb{R}$ é dita

1. continua se $\exists C$ tal que $|a(u, v)| \leq C|u||v| \forall u, v \in H$

2. coerciva se $\exists \alpha>0$ tal que $a(u, u) \geq \alpha|u|^{2} \forall u \in H$

3. simétrica se $a(u, v)=a(v, u)$

Teorema 3.2 (Lax-Milgram) Seja a $: H \times H \rightarrow \mathbb{R}$ uma forma bilinear contínua e coerciva e $f: H \rightarrow \mathbb{R}$ um funcional linear limitado. Então existe um único elemento $u \in H$ tal que,

$$
a(u, v)=\langle f, v\rangle \quad \forall v \in H
$$

Prova. Pelo teorema da representação de Riesz (ver teorema $3.2(3)$ ) existe um único $w \in H$ tal que $\langle f, v\rangle=(w, v)$ de tal modo que,

$$
a(u, v)=(w, v) \quad \forall v \in H .
$$

Agora, seja $A: H \rightarrow H$ e escrevendo $w=A u$ nota-se que pela continuidade de $a$,

$$
a(u, A u) \leq C\|A u\|_{H}\|u\|_{H},
$$

$\operatorname{como}(A u, A u)=a(u, A u)$,

$$
\|A u\|_{H}^{2}=(u, A u) \leq C\|A u\|_{H}\|u\|_{H} \forall u \in H .
$$


Deste modo $A$ é limitado, com $\|A u\| \leq C\|u\|$. Seja $\alpha, \beta \in \mathbb{R}$ e $u, w \in H$ para todo $v \in H$ vale,

$$
(A(\alpha u+\beta w), v)=a(\alpha u+\beta w, v)
$$

como $a(\alpha u+\beta w, v)=\alpha a(u, v)+\beta a(w, v)$ tem-se,

$$
(A(\alpha u+\beta w), v)=(\alpha A u+\beta w, v)
$$

logo, $A$ é limitado e linear. Notando que $A$ é injetivo uma vez que dada a coercividade e a continuidade de $a$ tem-se que,

$$
\begin{gathered}
a(u, u)=(A u, u) \leq C\|A u\|_{H}\|u\|_{H}, \\
\alpha\|u\|_{H}^{2} \leq a(u, u)=(A u, u),
\end{gathered}
$$

$\log \mathrm{O}$

$$
\beta\|u\|_{H} \leq\|A u\|_{H}
$$

de tal modo que $N(A)=\{0\}$, o que implica em $R(A)$ ser fechado (ver teorema 2.21 (2)) pelo fato . Notando que se $w \in H$ e $w \in R(A)^{\perp}$.

$$
(w, u)=0 \forall u \in R(A),
$$

uma vez que $A w \in R(A)$ tem-se que,

$$
\begin{gathered}
(w, A w)=0=a(A w, w), \\
\alpha\|w\|_{H}^{2} \leq(A w, w)=0,
\end{gathered}
$$

$\operatorname{assim} R(A)^{\perp}=\{0\}, \operatorname{logo} R(A)=H$. Como $A$ é bijetivo pode-se escrever $w=A u$ para $w \in H$ e $u \in H$. Novamente pelo teorema de Riesz tem-se um único $w \in H$ tal que,

$$
\langle f, v\rangle=(w, v) \quad \forall v \in H,
$$

assim, $a(u, v)=(A u, v)=\langle f, v\rangle$.

\section{2}

\section{Equações}

Uma vez desenvolvido o teorema de Lax-Milgram, pode-se partir para o estudo do objetivo desse trabalho, estudar a existência de soluções fracas para equações diferenciais de segunda ordem. 
Pode-se estudar a equação de Laplace com condição de contorno de Dirichlet, da forma,

$$
\begin{cases}-\Delta u=f & \text { em } \Omega, \\ u=0 & \text { em } \partial \Omega .\end{cases}
$$

Como (3-3) pode ser integrada por partes, pode-se reescrever o problema na forma fraca.

Definição 3.3 Chama-se forma fraca da equação de Poisson o problema tal que $\forall \phi \in C_{c}^{1}(\Omega)$,

$$
\int_{\Omega} D u D \phi=\int_{\Omega} f \phi
$$

Reparando que essa definição faz sentido uma vez que caso $u \in C^{2}(\Omega)$ (3-3) é equivalente a (3-4).

Uma vez definido o problema definimos os termos soluções fracas e fortes.

Definição 3.4 Chama-se de solução

- forte da equação de Poisson (3-3) $u \in C^{2}(\Omega)$ tal que u satisfaz (3-3),

- fraca da equação de Poisson (3-3) $u \in H_{0}^{1}(\Omega)$ que u satisfaz (3-4).

Definidas as classificações, pode-se tratar do problema de existência e unicidade para 3-4.

Teorema 3.5 Existe um único $u \in H_{0}^{1}(\Omega)$ tal que u satisfaz (3-4).

Prova. Definindo $a(u, v)=\int_{\Omega} D u D v d x$, pode-se provar que a é uma forma bilinear contínua e coerciva. Se $u, v \in H_{0}^{1}(\Omega)$ então por Hölder,

$$
a(u, v)=\int_{\Omega} D u D v d x \leq\|u\|_{2}\|v\|_{2}
$$

uma vez que $\|u\|_{2}\|v\|_{2} \leq\left(\|u\|_{2}+\|D u\|_{2}\right)\left(\|v\|_{2}+\|D v\|_{2}\right)$ tem-se,

$$
a(u, v)=\int_{\Omega} D u D v d x \leq\|u\|_{H^{1}}\|v\|_{H^{1}}
$$

tendo assim a continuidade de $a$. Usando a desigualdade de Poincaré tem-se,

$$
a(u, u)=\int_{\Omega}|D u|^{2} d x \geq C\|u\|_{2}^{2},
$$

usando a equivalência das normas tem-se,

$$
a(u, u) \geq C\|u\|_{H^{1}}^{2},
$$


tendo a coercividade de $a$.

Agora, aplicando o teorema de Lax-Milgram com $H=H_{0}^{1}(\Omega)$ e forma bilinear $a$, tem-se que existe um único $u \in H_{0}^{1}(\Omega)$ tal que $u$ satisfaz (3-4).

Como observado no capitulo 1 , caso $A_{i j}$ seja $C^{1}(\Omega)(1-2)$ pode ser escrito como (1-3) porem, se $A_{i j} \in L^{\infty}(\Omega)$ tal estratégia não pode ser usada. Para o estudo do problema a seguir fica subentendido que índices repetidos implicam em somas, assim,

$$
D_{i}\left(A_{i j} D_{j} u\right)=\sum_{i=1}^{n} \sum_{j=1}^{n} D_{i}\left(A_{i j} D_{j} u\right) .
$$

O problema pode ser formulado como,

e tem sua forma fraca,

$$
\begin{cases}-D_{i}\left(A_{i j} D_{j} u\right)=f & \text { em } \Omega, \\ u=0 & \text { em } \partial \Omega,\end{cases}
$$

$$
\begin{cases}\int_{\Omega} A_{i j} D_{j} u D_{i} \phi d x=\int_{\Omega} f \phi d x & \text { em } \Omega \\ u=0 & \text { em } \partial \Omega\end{cases}
$$

para todo $\phi \in H^{1}(\Omega)$.

Assim, tem-se o seguinte teorema.

Teorema 3.6 Para $A_{i j} \in L^{\infty}(\Omega)$ elíptico e limitado, ou seja, para algum $\lambda, \Lambda>0$ vale,

$$
\lambda|\xi|^{2} \leq A_{i j}(x) \xi_{i} \xi_{j} \leq \Lambda|\xi|^{2}, \forall x \in \Omega,
$$

para todo $\xi \in \mathbb{R}^{n}$ e $f \in L^{2}(\Omega)$, existe um único $u \in H_{0}^{1}(\Omega)$ tal que u é solução de (3-6).

Prova. Começa-se definindo a forma bilinear,

$$
a(u, v)=\int_{\Omega} A_{i j} D_{j} u D_{i} v d x
$$

Verificando que, $a$ é coerciva pelo fato da elipticidade de $A_{i j}$,

$$
a(u, u) \geq \int_{\Omega} \lambda|D u|^{2} d x
$$

aplicando a desigualdade de Poincaré tem-se,

$$
a(u, u) \geq C\|u\|_{H^{1}} .
$$

Resta agora reescrever o problema na sua forma fraca, ou seja,

$$
\int_{\Omega} A_{i j} D_{j} u D_{i} \phi d x=\int_{\Omega} f \phi d x .
$$


Assim, tem-se,

$$
a(u, \phi)=\langle f, \phi\rangle
$$

Usando o teorema de Lax-Milgram com $H=H_{0}^{1}(\Omega)$ conclui a prova.

Até o momento, foram estudados problemas com condição de fronteira homogenia. Para o estudo dos problemas não homogêneos o teorema de Stampacchia, desenvolvido a seguir, ajudará no tratamento de condições de contorno não homogenias.

Teorema 3.7 (Stampacchia) Seja a uma forma bilinear, contínua e coerciva em $H$. Seja $K \in H$ um subespaço fechado e convexo não vazio. Então, para qualquer $v \in H^{*}$, existe um único elemento $u \in K$ tal que,

$$
a(u, \phi-u) \geq\langle v, \phi-u\rangle, \forall \phi \in K \text {. }
$$

Ainda, se a também for simétrica, então u é caracterizado pela propriedade,

$$
\frac{1}{2} a(u, u)-\langle v, u\rangle=\min _{\phi \in K}\left\{\frac{1}{2} a(\phi, \phi)-\langle v, \phi\rangle\right\}
$$

Prova. Pelo teorema de Riesz, sabe-se que existe um único $f \in H^{*}$ tal que,

$$
\langle v, \phi\rangle=(f, \phi), \forall \phi \in H
$$

Para um $u$ fixo, a aplicação que leva de $\phi$ em $a(u, \phi)$ é um funcional linear contínuo em $H$. Usando, novamente, o teorema de Riesz existe um único elemento em $H$, denotado por $A u$ tal que,

$$
a(u, \phi)=(A u, \phi), \forall \phi \in H \text {. }
$$

Tem-se a continuidade e coercividade da forma bilinear $a$, uma vez que,

$$
\begin{gathered}
a(u, A u)=(A u, A u)=\|A u\|^{2} \leq C\|A u\|\|u\|, \\
\|A u\| \leq C\|u\|, \forall u \in H,
\end{gathered}
$$

e,

$$
a(u, u)=(A u, u) \geq \alpha\|u\|^{2}, \forall u \in H .
$$

Observando que 3-7 pode ser reescrito da forma,

$$
(A u, \phi-u) \geq(f, \phi-u), \forall \phi \in K \text {. }
$$

Seja $\rho>0$,

$$
(\rho f-\rho A u+u-u, \phi-u) \leq 0,
$$


é equivalente a 3-10, uma vez que,

$$
\begin{gathered}
\rho[(f, \phi-u)-(A u, \phi-u)]+(u, \phi-u)-(u, \phi-u) \leq 0, \\
(A u, \phi-u) \geq(f, \phi-u) .
\end{gathered}
$$

Deste modo, $u$ pode ser expresso como a projeção de $(\rho f-\rho A u+u)$ em $K$ (ver teorema $5.2(2))$.

Assim, escrevendo $u=P_{K}(\rho f-\rho A u+u)$, para todo $\phi \in K$, defini-se $S v=P_{K}(\rho f-\rho A v+v)$. Como $P_{K}$ não aumenta a distância (ver teorema 5.3 (2)) tem-se,

$$
\left\|S v_{1}-S v_{2}\right\|=\| P_{K}\left(v_{1}-v_{2}-\rho\left(A v_{1}-A v_{2}\right) \| .\right.
$$

Como a projeção tem normal menor ou igual ao objeto projetado,

$$
\left\|S v_{1}-S v_{2}\right\| \leq\left\|v_{1}-v_{2}-\rho\left(A v_{1}-A v_{2}\right)\right\| .
$$

Lembrando que $\|u\|^{2}=(u, u)$,

$$
\left\|S v_{1}-S v_{2}\right\|^{2}=\left\|v_{1}-v_{2}\right\|-2 \rho\left(A v_{1}-A v_{2}, v_{1}-v_{2}\right)+\rho\left\|v_{1}-v_{2}\right\|^{2}
$$

usando que $\left(A v_{1}-A v_{2}, v_{1}-v_{2}\right) \geq \alpha\left\|v_{1}-v_{2}\right\|^{2}$ e $C\left\|v_{1}-v_{2}\right\| \geq\|A u\|$, tem-se,

$$
\left\|S v_{1}-S v_{2}\right\|^{2} \leq\left\|v_{1}-v_{2}\right\|^{2}\left(1-2 \rho \alpha+\rho^{2} C^{2}\right)
$$

Uma vez que $\rho$ não foi fixado, pode-se tomar $k^{2}=1-2 \rho \alpha+\rho^{2} C^{2}$ de tal maneira que $\rho C^{2}-2 \alpha<0, \log \rho \rho$ é tal que $0<\rho<\frac{2 \alpha}{C^{2}}$. Assim, tem-se,

$$
\left\|S v_{1}-S v_{2}\right\| \leq k\left\|v_{1}-v_{2}\right\|, \operatorname{com} k<1
$$

usando o teorema do ponto fixo de Banach (ver teorema 1.A (6)), $S$ tem um único ponto fixo.

Assumindo que $a$ é também simétrica, $a$ define um novo produto interno (ver pg $720(4))$ em $H$, logo, $|a(u, u)|^{\frac{1}{2}}$ é uma nova norma e uma vez que,

$$
a(u, u)=(A u, u) \geq \alpha\|u\|_{H}^{2},
$$

e,

$$
|a(u, u)|^{\frac{1}{2}} \leq C\|u\|_{H},
$$

as normas são equivalentes.

Usando o teorema de Riez podemos representar o funcional $v$ com o novo 
produto interno, ou seja, existe um único $g \in H$ tal que,

$$
\langle v, \phi\rangle=a(g, \phi), \forall \phi \in H
$$

Logo, o problema 3-7 pode ser reescrito como,

$$
a(g-u, \phi-u) \leq 0, \forall \phi \in K
$$

Desse modo, $u$ é a projeção de $g$ em $K$ para o novo produto interno $a$, também tem-se que $u$ é o único em $K$ tal que,

$$
\min _{\phi \in K} a(g-\phi, g-\phi)^{\frac{1}{2}}
$$

é atingido (ver teorema 5.2 ](2)). Tem-se assim,

$$
\begin{gathered}
a(g-\phi, g-\phi)=a(g, g-\phi)-a(\phi, g-\phi), \\
a(g-\phi, g-\phi)=a(g, \phi)-2 a(g, \phi)+a(g, g) .
\end{gathered}
$$

Lembrando que $a(g, \phi)=\langle v, \phi\rangle$,

$$
a(g-\phi, g-\phi)=a(\phi, \phi)-2\langle v, \phi\rangle+a(g, g) \text {. }
$$

Isso é minimizar a quantidade,

$$
\frac{1}{2} a(\phi, \phi)-\langle v, \phi\rangle
$$

Agora, com o teorema de Stampacchia desenvolvido iremos estudar o seguinte problema,

$$
\begin{cases}-\Delta u+u=f & \text { em } \Omega, \\ u=g & \text { em } \partial \Omega,\end{cases}
$$

com $f \in L^{2}(\Omega)$ e $g \in H^{1}(\partial \Omega)$.

O problema pode ser reescrito, em sua forma fraca, como,

$$
\int_{\Omega} D u D \phi+u \phi d x=\int_{\Omega} f \phi d x, \forall \phi \in H_{0}^{1}(\Omega) .
$$

Teorema 3.8 Seja $\tilde{g} \in H^{1}(\Omega) \cap C(\bar{\Omega})$ tal que $\tilde{g}=g$ em $\partial \Omega$,

$$
K=\left\{\phi \in H^{1}(\Omega) ; \phi-\tilde{g} \in H_{0}^{1}(\Omega)\right\}
$$


Então, existe um único $u \in K$ tal que u minimiza,

$$
J(v)=\frac{1}{2} a(v, v)-\langle\phi, v\rangle .
$$

Prova. O conjunto $K$ é convexo uma vez que para todo $u, v \in K$ e $t \in \mathbb{R}$ tem-se que $t u+(1-t) v \in K$, e fechado uma vez que toda subsequência converge para um elemento de $K$.

Por (3.7) existe um único $u \in K$ tal que,

$$
\int_{\Omega} D u(D \phi-D u)+u(\phi-u)-f(\phi-u) d x \geq 0, \forall \phi \in H_{0}^{1}(\Omega) .
$$

Escolhendo $\phi=u \pm w \operatorname{com} w \in H_{0}^{1}(\Omega) \operatorname{com}$,

$$
a(u, \phi)=\int_{\Omega} D u D \phi+u \phi d x
$$

tem-se,

$$
a(u, w) \geq\langle f, w\rangle
$$

$\mathrm{e}$,

$$
a(u, w) \leq\langle f, w\rangle
$$

Como $u$ minimiza $J$, então, para todo $\phi \in H_{0}^{1}(\Omega)$ e $t \in \mathbb{R}$ vale,

$$
\begin{gathered}
J(u) \leq J(u+t \phi) \\
\frac{1}{2} \int_{\Omega} D u^{2}+u^{2}-f u d x \leq \frac{1}{2} \int_{\Omega} D(u+t \phi)^{2}+(u+t \phi)^{2}-f(u+t \phi) d x \\
0 \leq t^{2} \int_{\Omega} D \phi^{2}+\phi^{2} d x+t \int_{\Omega} D u D \phi+u \phi-f \phi d x
\end{gathered}
$$

dividindo por $t$ e fazendo $t \rightarrow 0$ com $t>0$ e $t<0$ resultará em,

$$
\int_{\Omega} D u D \phi+u \phi-f \phi d x=0
$$

como $\phi$ é arbitrário tem-se o resultado.

Com esses exemplos pode-se ver que a forma da equação não altera o procedimento a ser usado. 


\section{Referências bibliográficas}

[1] ADAMS, R.A.. Sobolev Spaces. Marcel Dekker, New York (NY, USA), 2nd edition, 2003.

[2] BREZIS, H.. Functional Analysis, Sobolev Spaces and Partial Differential Equations. Springer-Verlag New York, 1 edition, 2011.

[3] CONWAY, J.B. . A Course in Functional Analysis. Springer-Verlag New York, 2 edition, 2007.

[4] EVANS, L.C. . Partial Differential Equations. American Mathematical Society, 2 edition, 2010.

[5] GILBARD, D, TRUDINGER, N S.. Elliptic Partial Differential Equations of Second Order. Springer-Verlag Berlin Heidelberg, 2 edition, 2001.

[6] ZEIDLER, E.. Applied Functional Analysis. Springer-Verlag New York, 1 edition, 1995. 
A

\section{Desigualdades}

Teorema A.1 (Jensen) Seja $f \in L^{1}(\Omega)$ e $\mu: \Omega \rightarrow \mathbb{R}$ uma função convexa, então

$$
\mu\left(\int_{\Omega} f d x\right) \leq \int_{\Omega} \mu(f) d x
$$

Teorema A.2 (Minkowski) Se $1 \leq p \leq \infty$ e u,v $\in L^{p}(\Omega)$, então

$$
\|u+v\|_{p} \leq\|u\|_{p}+\|v\|_{p}
$$

Teorema A.3 (Interpolação) Seja $f \in L^{p} \cap L^{q}$ com $1 \leq p \leq q \leq \infty e$ $p \leq r \leq q$

$$
\|f\|_{r} \leq\|f\|_{p}^{\alpha}\|f\|_{q}^{1-\alpha}
$$

onde $\frac{1}{r}=\frac{\alpha}{p}+\frac{1-\alpha}{q}$ e $0 \leq \alpha \leq 1$.

Teorema A.4 (Hölder) Seja $1 \leq p_{1}, \ldots, p_{m}, \leq \infty$ com $\sum_{i=1}^{n} \frac{1}{p_{i}}=1$, se $u_{i} \in L^{p_{i}}(\Omega)$ para $i=1, \ldots, n$, então

$$
\int_{\Omega}\left|u_{1} \ldots u_{n}\right| d x \leq \prod_{i=1}^{n}\left\|u_{i}\right\|_{p_{i}}
$$

Teorema A.5 (Young) Seja $1<p, q<\infty \operatorname{com} \frac{1}{p}+\frac{1}{q}=1$, então

$$
a b \leq \frac{a^{p}}{p}+\frac{b^{q}}{q}
$$

para $a, b>0$ 